\title{
Archéopages
}

Archéopages

Archéologie et société

Hors-série 1 | 2008

Construction $^{s}$ de l'archéologie

\section{Archéologie et langage : éloge du scepticisme}

\section{Colin Renfrew}

\section{(2) OpenEdition}

\section{Journals}

Édition électronique

URL : https://journals.openedition.org/archeopages/867

DOI : 10.4000/archeopages.867

ISSN : 2269-9872

\section{Éditeur}

INRAP - Institut national de recherches archéologiques préventives

Édition imprimée

Date de publication : 1 février 2008

Pagination : 94-95

ISSN : 1622-8545

\section{Référence électronique}

Colin Renfrew, «Archéologie et langage : éloge du scepticisme », Archéopages [En ligne], Hors-série 1 | 2008, mis en ligne le 01 février 2008, consulté le 28 février 2023. URL : http://

journals.openedition.org/archeopages/867 ; DOI : https://doi.org/10.4000/archeopages.867 
LEMONNIER P. 1999: «Femmes et richesses en Nouvelle-Guinée», in P.Descola, J.Hamel, P. Lemonnier (dir), La Production du social. Autour de Maurice Godelier, Paris, Fayard, p. 315-332.

LÉVI-STRAUSS Cl. 1943: " Guerre et commerce chez les Indiens de l'Amérique du Sud », Renaissance, nº 1, p.122-139.

Lipuma E. 1988: The Gift of Kinship, Cambridge, Cambridge University Press.

Meggitt M. 1977: Blood is their Argument, Palo Alto, Mayfield Publishing Company.

SAHLINS M. 1963 : «Poor man, rich man, big-man, chief: political types in Melanesia and Polynesia ", Comparative studies in society and history, $\mathrm{n}^{\circ} 5$ (3), p. 285-303.

SERPENTI L. 1977: Cultivators in the Swamps. Social structure and horticulture in a New Guinea society (Frederik-Hendrik Island West New Guinea), Assen, Amsterdam, van Gorgum.

Williams F. E. 1936: Papuans of the Trans-Fly, Oxford, Clarendon Press.

\section{Archéologie et langage : éloge du scepticisme}

Colin Renfrew

McDonald Institute for Archaeological Research, Cambridge

$\mathrm{L}$ es spécialistes des études indo-européennes des années 1960-1970 affirmaient (ou présumaient) qu'au début de l'âge du Bronze, en Europe centrale et occidentale, une «arrivée » d'Indo-Européens avait eu lieu, de même qu'à une époque indéterminée de lâge du Fer avait eu lieu une «arrivée » des Celtes, et que durant l'âge du Bronze ou vers sa fin avait eu lieu également une «arrivée» des Grecs. Chacun de ces événements avait été d'abord avancé ou supposé sur une base linguistique, à charge pour l'archéologie d'en apporter ensuite la preuve.

Je souhaiterais souligner ici la position courageuse - solitaire parfois - de Jean-Paul Demoule $(1980,1999)$ sur les langues et les populations indo-européennes. C'est en archéologue qu'il aborda l'enjeu d'une possible réconciliation des données linguistiques et archéologiques en Europe. Il a clairement perçu que l'ensemble des opinions qui prévalaient dans les années 1960 et 1970, à l'instar de la plupart des précédentes, étaient sujettes à controverse car elles se fondaient sur des généralisations et des hypothèses sur les migrations qui nétaient plus appuyées par les preuves archéologiques. À l'époque, de son côté comme du mien, nous pouvions observer que les équations trop faciles entre des langues et des «cultures» archéologiques spécifiques, d'une part, et des «peuples» hypothétiques (souvent fictifs), d'autre part, qui étaient supposés avoir parlé ces langues et avoir créé ces cultures, présentaient d'irrémédiables défaillances.

Ma propre attitude à l'égard de cette constatation était peut-être moins politique, moins socialement engagée que la sienne (bien que nous partagions la même antipathie pour l'idéologie nazie, le racisme, et plus particulièrement l'antisémitisme); il en sait plus que moi sur l'histoire intellectuelle du $\mathrm{xx}^{\mathrm{e}}$ siècle et peut-être aussi sur les sympathies de certains intellectuels renommés, comme Georges Dumézil, dont nous rejetons d'un commun accord les synthèses. En ce qui me concerne, mon doute initial à propos des études indo-européennes des années 1960 et 1970 ne reposait pas tant sur une position politique que sur l'influence constante qu'elles exerçaient sur la reconstruction archéologique. En dépit de l'affection et du respect que je voue à Marija Gimbutas, sa reformulation de la théorie avancée un demi-siècle auparavant par Gordon Childe (qui n'était lui-même pas le premier) à propos d'invasions de guerriers nomades montés à cheval en provenance des steppes orientales m'a toujours paru manquer de substance archéologique. Or, cette hypothèse «kourgane» jouit encore plus ou moins des faveurs de la majorité des linguistes historiques et d'archéologues aussi réputés que James Mallory. Jean-Paul Demoule et moi-même pourrions probablement exprimer une critique identique à l'encontre de cette théorie - critique d'autant plus fondée que nous possédons à présent les preuves que le cheval ne fut utilisé pour la première fois dans des opérations militaires pour tracter des chars quà partir de 2000 environ avant notre ère, et qu'il y a peu d'éléments prouvant l'apparition d'une cavalerie montée avant le premier millénaire précédant notre ère.

Ces constatations ont amené Demoule à faire preuve d'audace et à rejeter l'intégralité du concept d'une langue proto-indo-européenne, une Ursprache et, partant, d'une Urheimat. Rares sont ceux qui ont eu le courage d'adopter une position aussi radicale. Contester la totalité des études indo-européennes demande un sacré culot! Des préoccupations similaires mont encouragé à réexaminer le concept indo-européen dans son ensemble, bien qu'avec une attitude moins radicale. J'étais surpris par le consensus apparent entre les linguistes selon lequel les relations entre les langues qualifiées aujourd'hui d'«indo-européennes» ne pourraient s'expliquer que par une origine commune, une Ursprache, autrement dit une prétendue langue «proto-indoeuropéenne », comme l'avait suggéré sir William Jones il y a quelque deux cent cinquante ans. Cela impliquerait une sorte d'arbre généalogique linguistique, calqué sur le modèle proposé par A. Schleicher en 1863, suivant les principes de Charles Darwin. Dans mon ouvrage intitulé Archaeology and Language (L'Énigme indoeuropéenne: archéologie et langage), j’ai souligné les autres possibilités proposées par la théorie des «vagues » de Johannes Schmidt, qui, dès 1875, démontrait comment des langues voisines pouvaient s'influencer les unes les autres et se renouveler au moyen d'emprunts. Mais peu de linguistes ont osé suggérer que les langues appartenant à ce que l'on appelle généralement une «famille linguistique» pouvaient devoir l'ensemble de leurs traits communs familiaux à ce processus d'emprunts, comme l'a fait Demoule. Du reste, le seul linguiste à avoir appliqué cette hypothèse à la famille des langues indo-européennes, pour autant que je sache, fut Nicolas Troubetskoï en 1939. Il y a quelques années, quand j'ai mentionné ses travaux en Australie, sa ligne de pensée fut reprise avec énergie par Robert Dixon (1997), mais pas 
au point de rejeter la notion d'un groupe à l'origine d'une langue proto-indo-européenne qui aurait donné naissance aux «langues-filles».

Les études les plus récentes (Gray et Atkinson 2003) tendent à obéir au modèle phylogénétique, ou «arborescent». Précisons toutefois que l'on prend de plus en plus conscience des limites de ce modèle «arborescent», par trop simpliste; une approche en «réseau» semble de mieux en mieux admise, car elle propose une alternative aux hypothèses des analyses phylogénétiques (Heggarty 2006). Dans un futur proche, cette tendance pourrait bien prendre la direction souhaitée par Demoule.

Je n'ai qu'un seul reproche à faire à sa position et j'ai eu l'occasion de le lui exprimer de vive voix lors d'une fort agréable rencontre (La Saga des langues indo-européennes) que nous avions animée ensemble à la Cité des Sciences, en 2005. Il se résume au fait que Demoule, à mon avis, n'a pas développé ses idées plus en détail. Sa critique est admirable. Sa vision ne l'est pas moins: «Il faut donc imaginer que les contacts prolongés, pendant des millénaires, de centaines de groupes humains successifs dans l'espace eurasiatique ont évidemment créé, par rencontres, osmoses, emprunts, et parfois aussi conquêtes, les nombreux points de convergence constatés. Il faut donc abandonner ce modèle arborescent, si pauvre et si funeste, pour des hypothèses historiques infiniment plus riches et plus complexes» (Demoule 1999).

Comme esquisse, ce qui précède est remarquable. Mais on aimerait voir cette ébauche prendre forme, ce modèle s'élaborer, parvenir à établir des corrélations plus spécifiques entre les données linguistiques et archéologiques. Elle pourrait reposer sur la théorie des «vagues» de changements linguistiques et jouir du support de l'analyse des réseaux employée avec discernement, afin d'échapper à ces arborescences phylogénétiques qui servent de moule standard à tant d'approches actuelles. Voilà la tâche qui reste à accomplir. Dès que Jean-Paul Demoule se sera libéré de ses fort utiles mais aussi fort prenantes responsabilités actuelles, il pourra retrouver le terrain et peaufiner les détails d'une perspective qu'il a déjà clairement esquissée voilà bientôt trente ans. Sa détermination scientifique, politique et philosophique est, je le crois, intacte.

En reconnaissant tout l'élan et l'intérêt de ce qu'il a déjà apporté dans ce domaine, je dois avouer que l'éloquence de son plaidoyer ne m'a pas encore totalement convaincu. Il m'en faut davantage. J'attends donc avec impatience ses futures œuvres consacrées à cette fascinante et controversée question indo-européenne.

Demoule J.-P. 1980: «Les Indo-Européens ont-ils existé? », L'Histoire, $\mathrm{n}^{\circ}$ 28, p. 109-120.

Demoule J.-P. 1999: «Destin et usage des Indos-Européens », Mauvais temps, no 5, juillet (Éditions Syllepse), p.1-7.

Dixon R. M. W. 1997: The Rise and Fall of Languages, Cambridge, Cambridge University Press.

Gray R. D. et ATKINSON Q. D. 2003: «Language-tree divergence times support the Anatolian theory of Indo-European origin », Nature, $\mathrm{n}^{\circ} 426$, p. 435-439.
Heggarty P. 2006: «Interdisciplinary indiscipline? Can phylogenetic methods meaningfully be applied to language data - and to dating language? ", in P. Forster et C. Renfrew (dir.), Phylogenetic methods and the prehistory of languages, Cambridge, McDonald Institute, p. 183-194.

RENFREw C. 1987 : Archaeology and Language, the Puzzle of IndoEuropean Origins, Londres, Jonathan Cape.

Troubetzkoї N. S. 1939: «Gedanken über das Indogermanenproblem », Acta Linguistica, $\mathrm{n}^{\circ}$ I, p. 81-89, reprinted in A. Scherer (dir.), 1968, Die Urheimat der Indogermanen, Darmstadt, Wissenschaftliche Buchgesellschaft, p. 214-223.

\section{Transgressions et vocations : travail manuel et travail intellectuel en archéologie}

\author{
Nathan Schlanger \\ Inrap
}

$\mathrm{S}$ ous les platanes de la place du village, deux hommes s'attardent [Fig.1]. Celui en blanc, bien à l'aise sur sa chaise pliante, canne au genou et cigarette aux lèvres (ce n'est que vers la quarantaine qu'il a appris à fumer, avec des muletiers du Levante espagnol), écoute ce que lui glisse à l'oreille l'homme en noir, à ses côtés. Un photographe aussi est là, pour prendre le cliché et en confier le tirage (sur carte postale) à l'homme assis, qui l'annote et l'incorpore dans un de ses albums autobiographiques, aujourd'hui déposé aux archives du musée des Antiquités nationales à Saint-Germain-en-Laye.

C'est en retournant cette photo pour en déchiffrer la légende que ce moment de complicité partagée prend les allures d'une anomalie, révélatrice de ce qu'est véritablement l'archéologie. Quelles confidences échangent donc nos deux compères?

Le vieil ouvrier, qui a travaillé avec moi à Gargas en 1912, me raconte qu'il a lu dans Dumont d'Urville que des indigènes de certaines îles se coupent les phalanges en signe de deuil, $\mathcal{E}$ que cela doit expliquer les mains de Gargas à doigts coupés. Gargas 19. IX. 36

Compères? Même si l'abbé Henri Breuil car c'est bien lui, assis, membre de l'Institut et du Collège de France, pape de la Préhistoire au sommet de sa gloire - n'en fait pas état, il y a bien là une infraction manifeste: voilà un ouvrier, anonyme de surcroît, consultant des sources premières pour en énoncer des hypothèses ethnoarchéologiques! Car, chez nos indigènes à nous, du monde occidental, c'est plutôt une division rigide, économique, sociale, voire morale, qui prévaut entre le travail manuel et le travail intellectuel, entre les moyens de production et les œuvres de la raison, entre la matière et l'esprit - un dualisme qui serait même constitutif de notre condition moderne.

Sauf peut-être, justement, en archéologie. En y regardant de plus près, la démarcation entre les champs d'action et de compétence de l'ouvrier et du savant semble n'avoir jamais été si tranchée ou infranchissable. Au cours des siècles, 